\title{
Comparison of Ruin Probabilities in Compound Poisson Risk Model
}

\section{Dol Nath Khanal}

Mathematics Faculty, Otero Junior College, La Junta, USA

Email: Dol.Khanal@ojc.edu

How to cite this paper: Khanal, D.N. (2019) Comparison of Ruin Probabilities in Compound Poisson Risk Model. Open Journal of Statistics, 9, 41-47.

https://doi.org/10.4236/ojs.2019.91004

Received: December 28, 2018

Accepted: January 22, 2019

Published: January 25, 2019

Copyright $\odot 2019$ by author(s) and Scientific Research Publishing Inc. This work is licensed under the Creative Commons Attribution International License (CC BY 4.0).

http://creativecommons.org/licenses/by/4.0/

\begin{abstract}
Compound Poisson risk model has been simulated. It has started with exponential claim sizes. The simulations have checked for infinite ruin probabilities. An appropriate time window has been chosen to estimate and compare ruin probabilities. The infinite ruin probabilities of two-compound Poisson risk process have estimated and compared them with standard theoretical results.
\end{abstract}

\section{Keywords}

Compound Poisson Risk Model, Ruin Probabilities Comparison, Simulations, Theoretical Results

\section{Introduction}

The Compound Poisson risk model can be modeled by the quotation,

$$
U(t)=u+c t-\sum_{i=1}^{N(t)} X_{i}
$$

where $N(t)$ and $\left\{X_{i}\right\}$ are independent. $X_{i}$ : amount of ith claim, $i=1,2,3, \cdots$ and $\left\{X_{i}\right\} \underbrace{\text { iid }} X$. u: initial surplus at time $t=0 . c$. rate of premium income per unit time. An additional assumption: $c>\lambda E[X]$ and thus

$$
c=(1+\theta) \lambda E[X]
$$

where $\theta>0$, loading coefficient and $\lambda$ is the rate of compound Poisson Process.

In this paper, the theoretical assumptions are: 1) In a Compound Poisson model, the ruin probability with initial reserve satisfies,

$$
\Psi(0)=\frac{1}{1+\theta}[1]
$$

and 2) In a compound Poisson model with exponential claim,

$$
\Psi(u)=\frac{1}{1+\theta} \exp \left[-\frac{\theta u}{\mu(1+\theta)}\right][1]
$$


where $X \sim \exp (\mu)$ and $E[X]=\mu$ have estimated using simulation. The compound Poisson risk model has been estimated.

It has assumed that the probability of ruin at time window of $T=1000$ after 100,000 independent runs which has set just to make algorithm convenience. For computational convenience, it has chosen the rate $\lambda=0.5$, loading coefficient $\theta=0.1$, initial surplus $u=0$ and claim size which are exponentially distributed with mean 1 . After simulation, an approximate $95 \%$ confidence interval based on 1,000,000 independent runs, and the confidence interval of $\Psi(0)$ : [0.9083541, 0.9094819] has obtained.

It helped to claim that the simulation which has done so far has very high accuracy since it is approximately equal to theoretical value of 0.909 from above given condition.

It has repeated to construct more confidence interval for each $\Psi(u)=\left[\Psi_{L}(u), \Psi_{U}(u)\right]$.

The time window and independent runs are changed as required. It has also estimated infinite ruin probabilities of two-compound Poisson Process and compared them. Let $U^{X}(t)$ and $U^{Y}(t)$ be defined by following model,

$$
U^{X}(t)=u+c^{X} t-\sum_{i=1}^{N(t)} X_{i}
$$

and

$$
U^{Y}(t)=u+c^{Y} t-\sum_{i=1}^{N(t)} Y_{i}
$$

where

$$
\left\{X_{i}\right\} \underbrace{i i d} X \text { and }\left\{Y_{i}\right\} \underbrace{i i d} Y \text { and } i=1,2,3, \cdots
$$

where

$$
c^{X}=(1+\theta) \lambda E[X] \text { and } c^{Y}=(1+\theta) \lambda E[Y]
$$

For simulation purpose set

$$
X=\min (Z, d)
$$

and

$$
Y=\frac{1}{2} Z
$$

with

$$
Z \sim \exp (1)
$$

where $d$ is such that $E[X]=E[Y]$ if and only if

$$
\left(1-\mathrm{e}^{-d}\right)=\frac{1}{2}
$$

Then

$$
d=\ln 2
$$

Setting $X$ and $Y$ such that $X=f(Z)$ and $Y=g(Z)$. This setting is motivated from reinsurance problem. Let $Z$ is the original risk and $f, g$ are different reinsurance strategies. 
Gatto and Mosimann [2] have solved for four different approaches to compute probability of ruin of insurer compound Poisson surplus process with an additive Wiener perturbation. There is no ideal comparison of ruin probabilities which have supported by theoretical results (15), (16) and (27). In this paper, infinite ruin probabilities of two-compound Poisson risk process have estimated and compared them with standard theoretical results.

\section{Compound Poisson Risk Model}

Suppose that at time $t=0$, the insurer has an amount of money set aside for the portfolio. This amount of money is called the initial surplus and is denoted by $u$. We can further assume that throughout this work that $u \geq 0$. The insurer needs this initial surplus because the future premium income on its own may not be sufficient to cover the future claims. We are also ignoring the expenses. The insurer's surplus at any time $t>0$ is a random variable since its value depends on the claims experience up to time $t$. The insurer's surplus at time $t$ is denoted by $U(t)$. The compound Poisson risk model modeled by (1), where $\left\{X_{i}\right\} \underbrace{i i d} X$ is the amount of $i$-th claims with $i=1,2,3, \cdots, N(t)$ is the amount of claims generated by portfolio in time $[0, t]$ where $N(t)$ is a Poisson process. We have $N(t)$ and $X_{i}$ are independent. Again $u$ is initial surplus at time $t=0$. The ruin probability of compound Poisson surplus process is denoted by

$$
\Psi(u)=P\left[\inf _{t>0} U(t)<0\right]
$$

We have an additional assumption that $c>\lambda E[X]$. Then $c$ can be expressed as

$$
c=(1+\theta) \lambda E[X]
$$

where $\theta>0$ is a loading coefficient and $\lambda$ is the rate of compound Poisson process. If $c<\lambda E[X]$, the ruin occurs with probability 1 which is generally not interested by insurer [1]. Intuitively, that means, averagely the premium should exceed the claim rate on one unit time period. We have from Wald's Identity,

$$
E\left[\sum_{i=1}^{N(t)} X_{i}\right]=\lambda t E[X]
$$

which can be proved by conditioning $N(t)$.

$$
E\left[\sum_{i=1}^{N(t)} X_{i}\right]=E\left[E\left[\sum_{i=1}^{N(t)} X_{i} \mid N(t)\right]\right]
$$

Notice that,

$$
E\left[\sum_{i=1}^{N(t)} X_{i} \mid N(t)=n\right]=E\left[\sum_{i=1}^{n} X_{i} \mid N(t)=n\right]=E\left[\sum_{i=1}^{n} X_{i}\right]=n E[X]
$$

which implies that $E\left[\sum_{i=1}^{N(t)} X_{i} \mid N(t)\right]=N(t) E[X]$ and consequently,

$$
E\left[\sum_{i=1}^{N(t)} X_{i}\right]=E[N(t) E[X]]=E[N(t)] E[X]=\lambda t E[x]
$$

\section{Theoretical Results}

Theorem 3.1. In a compound Poisson model defined by (1), the ruin proba- 
bility with no initial reserve satisfies

$$
\Psi(0)=1 /(1+\theta) \quad[1]
$$

Theorem 3.2. In a compound Poisson model defined by (1) with $X \sim \exp (\mu)$

$$
\Psi(u)=1 /(1+\theta) \exp [-\theta u / \mu(1+\theta)][1]
$$

\section{Simulation}

The purpose of simulation is to estimate infinite ruin probabilities at $\Psi(0)$ and compare $\Psi(0)$ to its theoretical value defined by (15). The steps are repeated for different $u$ value to develop $\Psi(u)$.

First, it has focused on estimating $\Psi(u, \tau)$ using simulation. In each run, the process is simulated up to time $\tau$ and the result of the $i$-th simulation run $Z_{i}$ is set to 1 if ruin occurs at or before time $\tau$ and to 0 if this is not the case. After $n$ runs, the Monte Carlo estimator is given by,

$$
Z=1 / n \sum_{i=1}^{n} X_{i}
$$

The following algorithm has used to obtain of a single simulation run.

Step 1: Initialize the simulation: set $U(0)=u$.

Step 2: Draw an exponential inter-arrival time $I$ with parameter $\lambda$ and draw a claim size $X \sim F$.

Step 3: If $t+I>\tau$ return $Z_{i}=0$. If not, set $U=u+c I-X$ and if $U<0$ return $Z_{i}=1$. Set $t=t+I$. Otherwise, return to step 2 .

For computational convenience, it has assumed that the probability of ruin time at time window of $T=1000$ after 1,000,000 independent runs which are assigned just to make algorithm convenience. The parameters which are defined in (1) are chosen: $\lambda=0.5, \theta=0.1$ with initial surplus $u=0$ and claim size are exponentially distributed with mean 1 . An approximate $95 \%$ confidence interval of $\Psi(0)$ based on 1,000,000 independent runs, is given by [0.9083541, $0.9094819]$.

We expect to see that the theoretical curve lies in between the confidence intervals. The plot from above procedure has expected to see a good estimate. However, it is not the case always since the time window chosen may not be the sufficient to provide an accurate estimate. The simulation has repeated to see different confidence interval for each $\Psi(u)=\left[\Psi_{L}(u), \Psi_{U}(u)\right]$. Then four different ruin probabilities plots are estimated from simulation (Figure 1).

One main issue is that the Monte Carlo simulation used doesn't work for small ruin probabilities. It could be the reason the graph doesn't display the probabilities of ruin smaller than 0.15 . We need to test for more surplus with sufficient time window to address this issue. For this, important sampling or other method is required. But the scope of this paper is to extend this estimation to compare ruin probabilities $\Psi_{X}(u) \leq \Psi_{Y}(u)$ defined by theorem 8.1 which has demonstrated on Figure 2. 


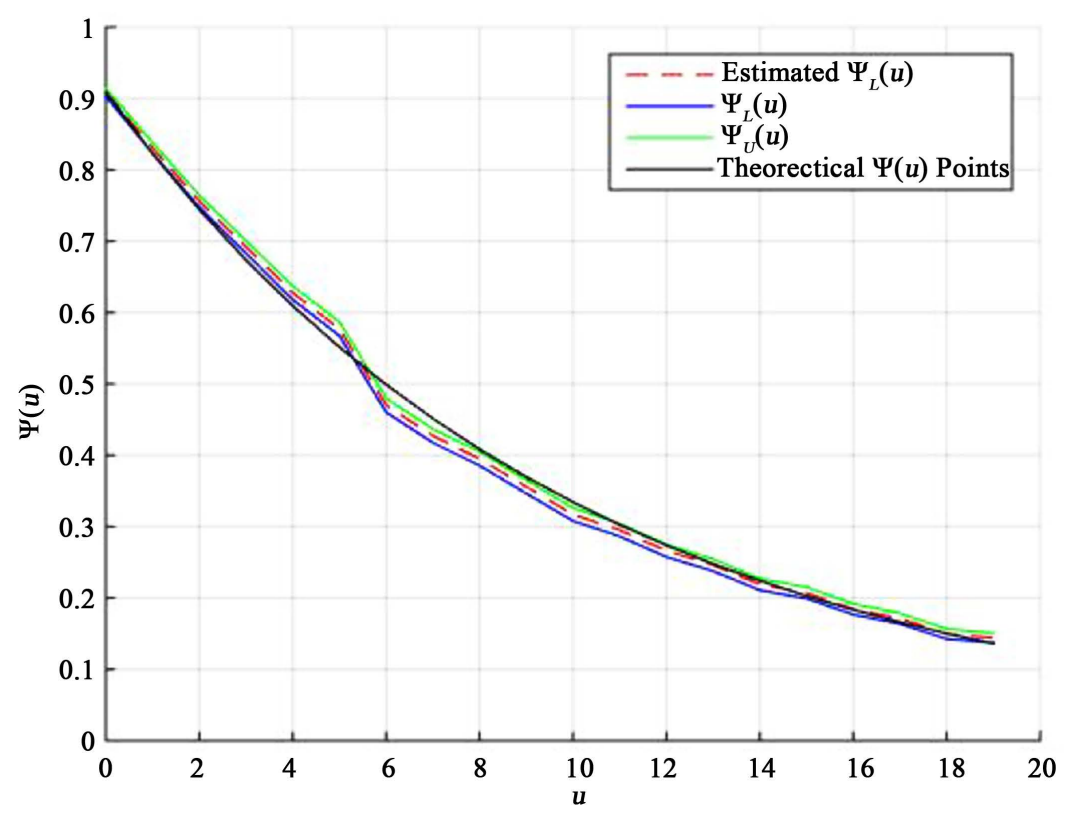

Figure 1. Ruin probabilities plot.

\section{Ruin Probabilities Comparison of Two Compound Poisson Model with Different Claims}

Definition: Let $X$ and $Y$ be two random variable where $X$ is smaller than $Y$ in convex order denoted by $X \leq_{c x} Y$ such that

$$
E[\phi(X)] \leq E[\phi(Y)]
$$

for all convex function $\phi: \mathbb{R} \rightarrow \mathbb{R}$, provided the expectation exists.

\section{Theoretical Results}

Theorem 6.1. For two random variable $X$ and $Y$ defined from (18)

$$
X \leq_{c x} Y \Rightarrow E[X]=E[Y]
$$

provided that the expectation exist [3].

Theorem 6.2. For two random variable $X$ and $Y$ defined from (18)

$$
X \leq_{c x} Y \Rightarrow \operatorname{Var}[X]=\operatorname{Var}[Y]
$$

provided that expectation exist [3].

The above result implies that $X \leq_{c x} Y$ means $Y$ has more dispersion. The above result is important because this guarantees that the premium rate,

$$
c^{X}=c^{Y}
$$

which makes easier to compare the ruin probabilities. A typical way to construct $X$ and $Y$ following order $\leq_{c x}$ has seen from Ohlin's Lemma.

\section{Ohlin's Lemma}

Lemma 7.1. Let $Z$ be a positive random variable and $f$ and $g$ be increasing functions. If $g$ crosses $f$ only once from below, i.e., there exist $x_{o}$, such that 


$$
f(x) \geq g(x) \text { for all } x<x_{o} \text {, and } E[f(Z)]=E[g(Z)]
$$

then $f(Z) \leq_{c x} g(Z)[4]$.

The above setting is motivated from reinsurance problem. We can think $Z$ as the original risk and $f, g$ are different insurance strategies. In this context, besides increasing, $f$ and $g$ are assumed to be continuous and satisfy $0 \leq f(x) \leq x$.

\section{Compound Poisson Risk Models with Different Claims}

It can be defined two compound Poisson risk model with different claims $U^{X}(t)$ by (5) and $U^{Y}(t)$ by (6). Again $c^{X}$ and $c^{Y}$ which are defined in (7) are respective rate of premium income per unit time of the model defined by (5) and (6). We are interested in two quantities: the ruin time and infinite ruin probability of two different models defined by (5) and (6) respectively.

$$
\begin{gathered}
\tau^{X}=\inf \left[t>0: U_{t}^{X}<0\right] \\
\Psi^{X}(u)=P\left(\tau^{X}<\infty\right)
\end{gathered}
$$

$\&$

$$
\begin{array}{r}
\tau^{Y}=\inf \left[t>0: U_{t}^{Y}<0\right] \\
\Psi^{Y}(u)=P\left(\tau^{Y}<\infty\right)
\end{array}
$$

Theorem 8.1. Let $U^{X}(t)$ and $U^{Y}(t)$ be defined in (4) and (5) respectively with generic claim size $X$ and $Y$. If $X \leq_{c x} Y$, then $\Psi_{X}(u) \leq \Psi_{Y}(u) \quad$ [3]. (27)

It has interested to see the above theoretical results by using simulation. The theoretical result has observed in simulation for different values. For simulation purpose we can set,

$$
X=Z \Lambda d
$$

and

$$
Y=1 / 2 Z
$$

with $Z \sim \exp (1)$ where such that

$$
E[X]=E[Y] \Leftrightarrow\left(1-\mathrm{e}^{-d}\right) 1 / 2
$$

Now compared with first simulation, $X$ becomes $f(Z)$ and $Y$ becomes $g(Z)$. From Ohlin's Lemma, we have $X \leq_{c x} Y$.

The practical background of

$$
X=Z \Lambda d
$$

is the excess of loss insurance form. For example, customer's loss in a car accident with an auto insurance where only the part in excess of certain level is covered by insurance company. The maximum loss for the customer has capped at d. Again the setting

$$
Y=1 / 2 Z
$$

is applicable on quota share (proportional insurance). Graph of $\Psi_{X}(u) \leq \Psi_{Y}(u)$ has estimated as Figure 2. 


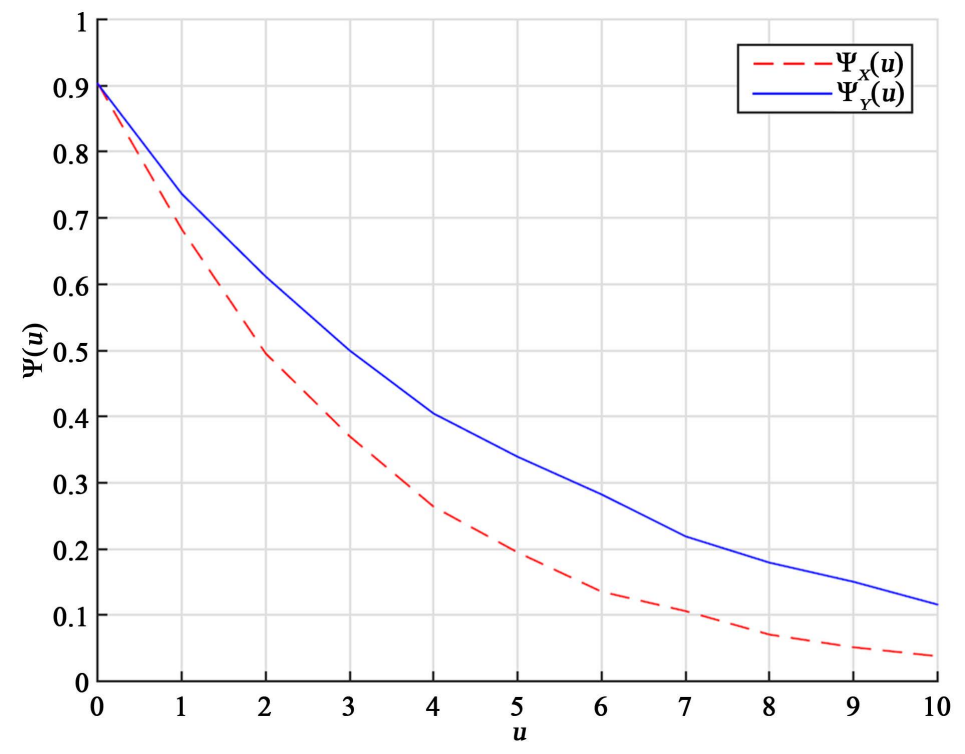

Figure 2. Ruin probabilities plot for $\Psi_{X}(u)$ and $\Psi_{Y}(u)$.

\section{Conclusion}

The infinite ruin probabilities of $\Psi(u)$ has been estimated and observed. The infinite ruin probability at $\Psi(0)$ has been observed by using simulation. It has found that $\Psi(0)$ is equal to its theoretical value, $\Psi(0)=\frac{1}{1+\theta}$. Then the comparison of $\Psi_{X}(u)$ and $\Psi_{Y}(u)$ has estimated for two-compound Poisson risk models. It has been verified that $\Psi_{X}(u) \leq \Psi_{Y}(u)$ for different $u$ values. Eventually, the theorem defined by (27) has been verified from estimations.

\section{Conflicts of Interest}

The author declares no conflicts of interest regarding the publication of this paper.

\section{References}

[1] Klugman, S., Panjer, H. and Willmot, G. (2008) Loss Model: From Data to Decision. 3rd Edition, Wiley, New York. https://doi.org/10.1002/9780470391341

[2] Gatto, R. and Mosimann, M. (2012) Four Approaches to Compute the Probability of Ruin in Compound. Poisson Risk Process with Diffusion. Mathematical and Computer Modelling, 55, 1169-1185. https://doi.org/10.1016/j.mcm.2011.09.041

[3] Shaked, M. and Shanthikumar, J.G. (2007) Stochastic Orders. Springer, New York. https://doi.org/10.1007/978-0-387-34675-5

[4] Asmuss, S. (2000) Ruin Probabilities. World Scientific Co. Pte. Ltd., Singapore. https://doi.org/10.1142/2779 